\title{
Wernicke's Encephalopathy: Typical Disease with an Atypical Clinicoradiological Manifestation
}

\author{
Rajesh Verma ${ }^{1}$ Chetan Kumar ${ }^{1}$
}

Address for correspondence Rajesh Verma, DM, DNB, Department of Neurology, King George's Medical University, Lucknow 226003 Uttar Pradesh, India (e-mail: drrajeshverma32@yahoo.com).

\begin{abstract}
Keywords

- Wernicke's encephalopathy

- ataxia

- ophthalmoplegia

- thiamine deficiency

Wernicke's encephalopathy is an acute neurological disorder caused due to thiamine deficiency. It is characterized by a triad of confusion, ataxia, and ophthalmoplegia. Wernicke's encephalopathy can present with atypical clinical manifestations, particularly in setting of nonalcoholic background. Our patient manifested with clinical complaints of acute drowsiness, speech difficulty, and ataxia. Magnetic resonance imaging of the brain revealed $\mathrm{T} 2$ hyperintensity in bilateral medial thalami, globus pallidus, periaqueductal gray matter, dorsal pons, and dorsal medulla. The diagnosis of Wernicke's encephalopathy was entertained and he well responded to parental thiamine therapy. The clinician should be aware about atypical presentation of Wernicke's encephalopathy.
\end{abstract}

\section{Introduction}

Wernicke's encephalopathy is an acute neurological syndrome caused due to thiamine deficiency. Clinically, it is characterized by a triad of confusion, ataxia, and ophthalmoplegia. ${ }^{1}$ The diagnosis is frequently missed especially in patients having atypical clinical manifestations, as shown by multiple autopsy studies, where in the diagnosis of Wernicke's encephalopathy is made after post mortem..$^{2,3}$ It occurs commonly in chronic alcoholics. It is also known to occur in the setting of anorexia, gastric bypass surgery, malignancies of the gastrointestinal tract, and total parenteral nutrition. In the rest, the disease can manifest itself in various ways based on the anatomical structures that get affected. Therefore, it is important to be aware of certain atypical clinical and radiological manifestations of the disease, so that early treatment can be initiated thereby ensuing good recovery and minimal residual deficits.

\section{Case Report}

A 60-year-old male, presented with acute history of speech difficulty and ataxia. The patient had been fasting for 22 days for religious reasons. Subsequently, the patient developed episodes of vomiting for 2 days followed by development of drowsiness, speech difficulty, and ataxia. It was not associated with fever/headache/seizures. The patient was subsequently admitted to a nearby hospital where he was managed with injectable medications (the details of which were not available with the patient). Three days later his sensorium improved. He was unable to speak and he developed ataxia.

The patient got discharged in the same state. The patient contacted our tertiary center for further management.

On examination, the patient was appeared listless and cachectic. His vital parameters were normal. Neurological examination revealed cerebellar ataxia, generalized rigidity, and finger-nose test was abnormal bilaterally. Dysdiadochokinesia was present on both sides. Severe anarthria was noted. His reflexes were intact and bilateral plantar reflex showed flexor response. No deficits were noted during examination of power. Deep tendon reflexes were intact. No signs of meningeal irritation were noted.

His complete blood count, renal function test, liver function test, thyroid profile, vitamin b12 levels, creatine phosphokinase (cpk) levels were within normal limits. Cerebrospinal fluid analysis/virology was normal. Electroencephalogram was unremarkable. Radiological examination of the chest and abdomen did not show any abnormality. Magnetic resonance 

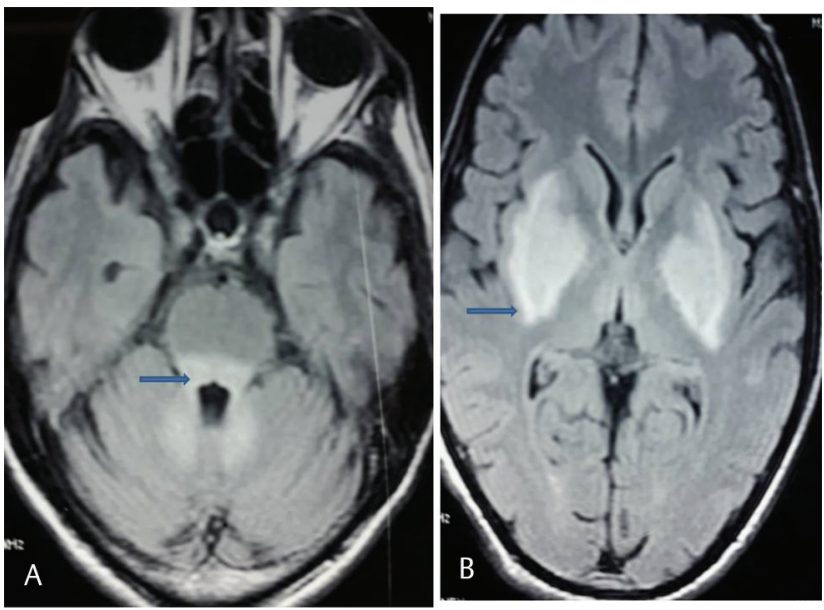

Fig. 1 Magnetic resonance imaging of cranium, (A) demonstrated periaqueductal gray matter and pontine hyperintense signal intensities (blue arrows), (B) revealed bilateral globus pallidus symmetrical hyperintense signals.

imaging (MRI) of the brain showed T2 hyperintensity in bilateral medial thalami, globus pallidus, periaqueductal gray matter, dorsal pons, and dorsal medulla (-Fig. 1).

Based on clinical and radiological findings, a diagnosis of Wernicke's encephalopathy was made. The patient was treated with high-dose thiamine ( $600 \mathrm{mg} /$ day). Gradually over a period of 2 weeks, there was improvement in his general condition. His speech improved and he was able to walk with assistance. His repeat MRI of the brain study revealed resolution of lesion ( - Fig. 2 ).

\section{Discussion}

Wernicke's encephalopathy is a medical emergency associated with high rates of morbidity and mortality without timely intervention. It was first described by Carl Wernicke in 1881 and its association with thiamine deficiency was made in the 1940s. Thiamine is an essential cofactor for metabolism of glucose as it plays an important role in the Kreb's cycle. Brain is solely dependent on glucose for nutrition, therefore deficiency of thiamine produces cellular energy deficit, focal acidosis, regional increase in glutamate, and ultimately cell death. ${ }^{4}$ The commonly affected areas include thalamus, mammillary bodies, periaqueductal and paraventricular areas, locus ceruleus, cranial nerves nuclei, and reticular formation.

In our patient, the presenting symptom was gross cerebellar ataxia and anarthria. It was not associated with ophthalmoplegia or alteration of sensorium. Only one of the clinical features of triad of Wernicke's encephalopathy was noted. The classically described triad of Wernicke's encephalopathy is noted only in $16 \%$ of patients with Wernicke's encephalopathy

The typical MRI features of Wernicke's encephalopathy includes symmetrical signal alterations in thalami, periaqueductal gray matter, mammillary bodies, and tectal plate. ${ }^{5}$ In our patient, apart from the typical areas, signal intensity changes were also noted in bilateral basal ganglia, dorsal pons,

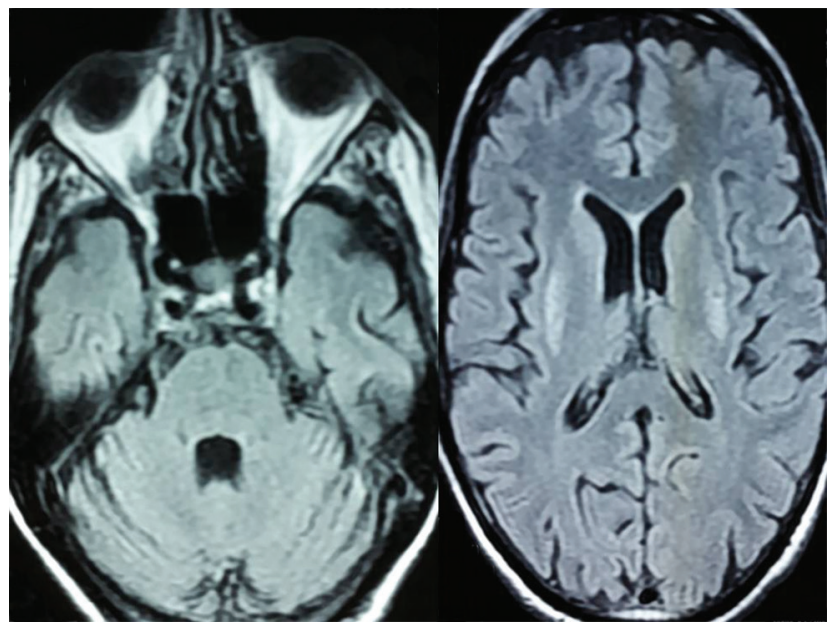

Fig. 2 Repeat MRI imaging of cranium after a month showed resolution of lesion. MRI, magnetic resonance imaging.

splenium, and cerebellar vermis which are relatively uncommon. ${ }^{5,6}$ The involvement of atypical areas in MRI is common among non alcoholic patients as was the case with our patient.

\section{Conclusion}

Our case aims to highlight the importance of suspecting Wernicke's encephalopathy in patients who do not fit into classically described clinical and radiological parameters. Most importantly early diagnosis and timely initiation of treatment leads to early recovery and lack of functional deficits.

\section{Funding}

None.

Conflict of Interest

None declared.

\section{References}

1 Wernicke C, Die akutehämorrhagischepolioencephalitis superior. Lehrbuch der GehirnkrankheitenfürÄrzte und Studierende; Vol. II. Kassel: Fischer Verlag; 1881:229-242

2 Harper CG, Giles M, Finlay-Jones R. Clinical signs in the Wernicke-Korsakoff complex: a retrospective analysis of 131 cases diagnosed at necropsy. J Neurol Neurosurg Psychiatry 1986;49(4):341-345

3 Harper C. The incidence of Wernicke's encephalopathy in Australia-a neuropathological study of 131 cases. J Neurol Neurosurg Psychiatry 1983;46(7):593-598

4 Thomson AD, Cook CC, Touquet R, Henry JA; Royal College of Physicians, London. The Royal College of Physicians report on alcohol: guidelines for managing Wernicke's encephalopathy in the accident and Emergency Department. Alcohol Alcohol 2002;37(6):513-521

5 Zuccoli G, Gallucci M, Capellades J, et al. Wernicke encephalopathy: MR findings at clinical presentation in twenty-six alcoholic and nonalcoholic patients. AJNR Am J Neuroradiol 2007;28(7):1328-1331

6 Suzuki S, Ichijo M, Fujii H, Matsuoka Y, Ogawa Y. Acute Wernicke's encephalopathy: comparison of magnetic resonance images and autopsy findings. Intern Med 1996;35(10):831-834 